\title{
Ponte miocárdica: revisão de literatura
}

\section{Myocardial bridging: literature review}

\author{
Éric Guimarães Machado', Ana Gabriela Menezes de Jesus Torres', Léo \\ Guimarães Soares ${ }^{2}$, Gabriel Porto Soares ${ }^{3}$, Paulo Sérgio Lopes Soares ${ }^{3}$
}

\begin{abstract}
Machado EG, Torres AGMJ, Soares LG, Soares GP, Soares PSL. Ponte miocárdica: revisão de literatura / Myocardial bridging: literature review. Rev Med (São Paulo). 2012;91(4):241-5.
\end{abstract}

\begin{abstract}
RESUMO: A ponte miocárdica é uma anomalia congênita das artérias coronárias em que feixes de miocárdio envolvem um segmento de artéria coronária epicárdica, levando a compressão de um segmento na sístole ventricular, se revertendo na diástole. Ela constitui um dos principais diagnósticos diferenciais de doença arterial coronariana, podendo se manifestar como dor torácica, infarto agudo do miocárdio ou morte súbita, mas na maioria das vezes é assintomática. Sua fisiopatologia ainda é controversa. Seu diagnóstico clínico deve ser considerado em pacientes com dor no peito e sem fatores de risco para doenças cardiovasculares. Nos exames complementares, o achado mais comum na cineangiocoronariografia é a compressão de um segmento coronário durante a sístole, revertido na diástole. A conduta de primeira linha no tratamento da ponte miocárdica sintomática é o uso de medicamentos para reduzir sintomas de isquemia e minimizar o risco de futuros eventos cardíacos. Na persistência da sintomatologia ao tratamento clínico, a intervenção percutânea com o uso de stents ou o tratamento cirúrgico estão indicados. O prognóstico a longo prazo em geral é bom. Embora seja um achado anatômico geralmente assintomático, em raros casos pode causar complicações importantes, inclusive morte súbita. Portanto, a ponte miocárdia deve ser considerada em pacientes jovens, com baixo risco para doenças cardiovasculares e sem evidências de isquemia miocárdica, porém com dor torácica ou sintomas não condizentes com a severidade da doença arterial coronariana.
\end{abstract}

DESCRITORES: Ponte miocárdica; Doença da artéria coronariana; Dor no peito; Literatura de revisão como assunto.
ABSTRACT: The myocardial bridging is a congenital anomaly of the coronary arteries in which bundles of myocardial involve a segment of the epicardial coronary artery, leading to compression of a segment in the ventricular systole, reversing itself during diastole. It's one of the main differential diagnosis of coronary artery disease and may manifest as chest pain, myocardial infarction or sudden death, but in most cases are asymptomatic. Its pathophysiology is still controversial. His clinical diagnosis should be considered in patients with chest pain and no risk factors for cardiovascular disease. In laboratory exams, the most common finding in coronary angiography is the compression of a coronary segment during systole, reversed during diastole. The first treatment of symptomatic myocardial bridging is medications to reduce symptoms of ischemia and minimize the risk of future cardiac events. In the persistence of clinical symptomatology, percutaneous intervention using stents or surgical treatment are types of threatment. The long-term prognosis is usually good. Although an anatomical finding is usually asymptomatic, in rare cases can cause major complications, including sudden death. Therefore, the myocardial bridge should be considered in young patients at low risk for cardiovascular disease and no evidence of myocardial ischemia, but with no chest pain or symptoms consistent with the severity of coronary artery disease.

KEYWORDS: Myocardial bridging; Coronary artery disease; Chest pain; Review literature as topic.

Trabalho realizado na Universidade Severino Sombra (USS), Vassouras, RJ, Brasil.

${ }^{1}$ Curso de Medicina - Universidade Severino Sombra (USS), Vassouras, RJ, Brasil.

2 Programa de Pós-graduação em Odontologia (Doutorado), Universidade do Estado do Rio de Janeiro (UERJ), Rio de Janeiro, RJ, Brasil.

${ }^{3}$ Setor de Cardiologia - Universidade Severino Sombra (USS), Vassouras, RJ, Brasil.

Endereço para correspondência: Éric Guimarães Machado. Rua Alfredo da Costa Mattos Jr., 75. Centro. Paraíba do Sul, RJ. CEP: 25850-000. e-mail: ericgmac@ hotmail.com 


\section{INTRODUÇÃO}

O trajeto intramiocárdico de uma artéria coronária epicárdica foi relatado pela primeira vez por Reyman, em $1737^{1}$, e demonstrado em angiografias em $1960^{2}$. Esta condição clínica foi denominada ponte miocárdica.

A ponte miocárdica ( $P M)$ é definida como uma anomalia congênita das artérias coronárias, em que feixes de miocárdio envolvem um segmento de artéria coronária epicárdica (Figura 1), levando a compressão de um segmento desta coronária durante a sístole ventricular, reversível na diástole ${ }^{2,3}$. Este achado anatômico também pode ser descrito como artéria coronária intramural, artéria coronária mural ou coronária mural ${ }^{4}$.

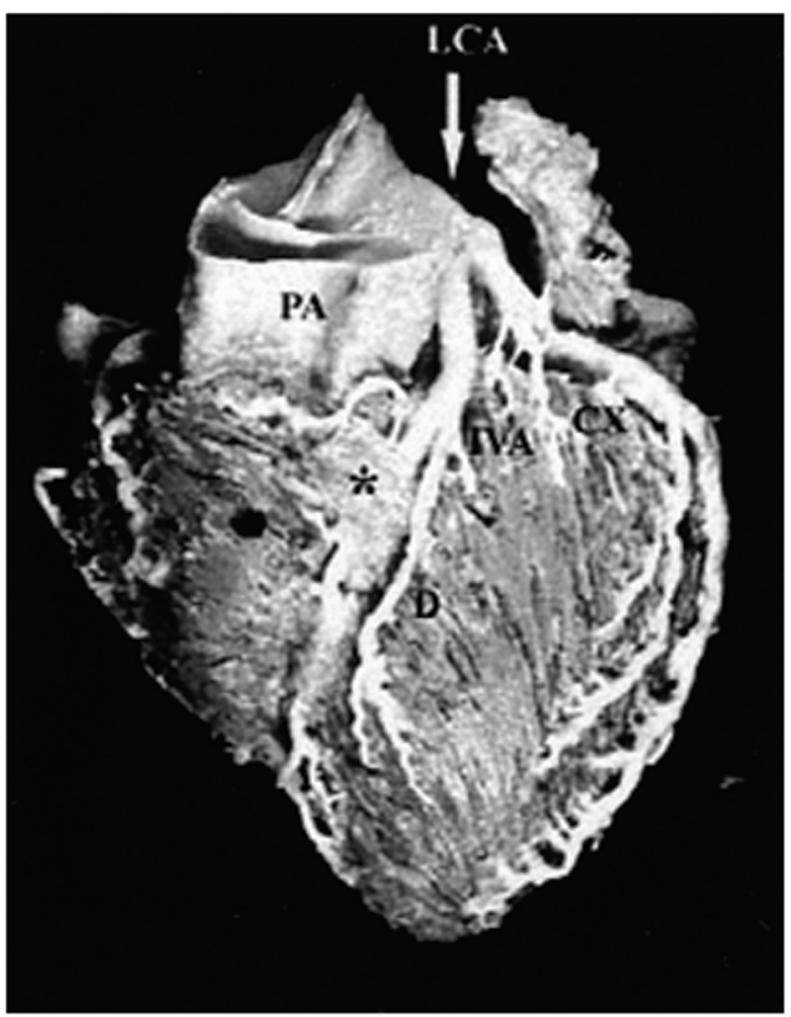

Figura 1. Ponte miocárdica: o terço médio da artéria interventricular anterior é envolvido por um segmento de miocárdio $\left.{ }^{*}\right)$. (CX: artéria circunflexa; D: artéria diagonal; IVA: artéria interventricular anterior; LCA: artéria coronária esquerda; PA: artéria pulmonar). (Fonte: Vilallonga ${ }^{15}$ )

A prevalência de PM em estudos angiográficos é de $1,7 \%$ (podendo variar entre $0,5 \%$ e $12 \%$ ), acometendo quase que na sua totalidade a artéria interventricular anterior ${ }^{2}$.

A PM faz diagnóstico diferencial com doença arterial coronariana, podendo ter como manifestação dor torácica típica ou atípica e até mesmo infarto agudo do miocárdio (IAM) e morte súbita ${ }^{3}$.
O objetivo do presente trabalho foi realizar uma revisão de literatura sobre ponte miocárdica, destacando sua apresentação clínica, exames complementares a serem realizados, diagnóstico e possibilidades terapêuticas.

\section{MÉTODOS}

Foi realizada uma revisão de literatura, entre os meses de janeiro a março de 2011, pesquisando os bancos de dados MEDLINE/PubMed e Lilacs/SciELO empregando os descritores "ponte miocárdica, doença da artéria coronariana e dor no peito".

\section{Manifestações clínicas}

A ponte miocárdica é assintomática na maioria dos pacientes ${ }^{5}$, porém pode se manifestar como angina pectoris, isquemia miocárdica, IAM, disfunção ventricular esquerda, fibrilação ventricular, bloqueio atriventricular paroxístico e morte súbita ${ }^{1,4-8}$. Porém, estas são complicações raras desta enfermidade ${ }^{1,9}$.

A intensidade dos sintomas depende de fatores como a quantidade, a espessura, a localização e o comprimento das PM, que são variáveis entre os pacientes ${ }^{4}$.

\section{Fisiopatologia e associação com aterosclerose}

A fisiopatologia da PM ainda é muito controversa ${ }^{3,7,10,11}$, no entanto, segundo Lima et al. ${ }^{4}$, alguns estudos sugerem que as PM são formadas junto com as artérias coronárias no período embrionário; outros defendem que a PM não é uma variação anatômica, mas um defeito na reabsorção destas fibras de músculos que envolve as coronárias epicárdicas.

Quanto a associação com aterosclerose, a área logo abaixo da PM é poupada pela doença aterosclerótica, no entanto, a área proximal à ponte é mais propensa a desenvolver aterosclerose. $\mathrm{O}$ desenvolvimento de aterosclerose no segmento proximal da PM pode ter relação com alterações no fluxo sanguíneo daquela área, fazendo as células endoteliais alterar a produção de substâncias vasoativas, influenciando em funções celulares vasculares como o controle do tônus vascular, do potencial trombogênico e da regulação do fluxo sanguíneo ${ }^{3}$.

\section{Diagnóstico clínico}

A PM deve ser investigada naqueles pacientes, em geral após a terceira década de vida, que apresentam dor anginosa, porém sem fatores de risco 
para doenças cardiovasculares ou sem evidências de isquemia ${ }^{3}$.

Segundo Pereira et al. ${ }^{3}$, a PM seria subdiagnosticada pelo fato de pequena parcela dos pacientes se apresentarem sintomáticos, somado a isto a indisponibilidade de métodos mais eficazes para identificação desta condição clínica.

\section{Exames complementares}

De acordo com Möhlenkamp et al. ${ }^{1}$, o eletrocardiograma de repouso frequentemente se apresenta com atividade elétrica normal; podem haver sinais inespecíficos de isquemia, distúrbios de condução ou arritmias cardíacas nos testes provocativos de estresse.

Na cintilografia miocárdica podem ser encontradas falhas na perfusão, porém este não é um achado obrigatório, até mesmo nas pontes profundas com grande compressão sistólica ${ }^{1}$.

A cineangiocoronariografia é o exame padrão ouro para diagnóstico da $\mathrm{PM}^{1}$. O achado mais comumente encontrado é a compressão de um segmento coronário durante a sístole, levando ao estreitamento, que se reverte na diástole ${ }^{3}$ (Figura 2). Avaliando sua natureza transitória e a dinâmica da obstrução, é possível fazer o diagnóstico diferencial com as estenoses coronarianas fixas. Caso as coronárias não apresentem anormalidades, podem ser utilizados testes provocativos, através da acentuação da compressão sistólica do segmento tunelizado ${ }^{3}$.

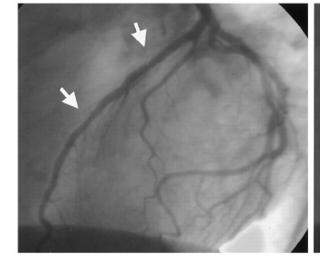

Diastole

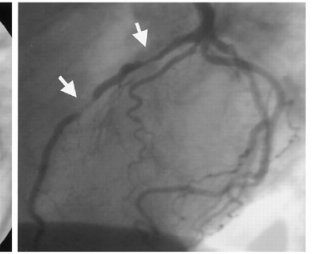

Systole
Figura 2. Arteriografia coronária: as setas brancas indicam a compressão sistólica típica da artéria interventricular anterior. Na diástole, a dimensão do lúmen da artéria se encontra sem alterações. Não há sinais angiográficos de aterosclerose coronariana. (Fonte: Möhlenkamp et al. ${ }^{1}$ )

Além disso, a angiotomografia computadorizada também é aceita como um método confiável e sensível a ser utilizado para o diagnóstico de $\mathrm{PM}^{12}$ (Figura 3), assim como a tomografia com coerência óptica, revelando se há presença de doença aterosclerótica e também estreitamento do vaso na sístole, com retorno de sua patência na diástole ventricular ${ }^{13}$ (Figura 4).

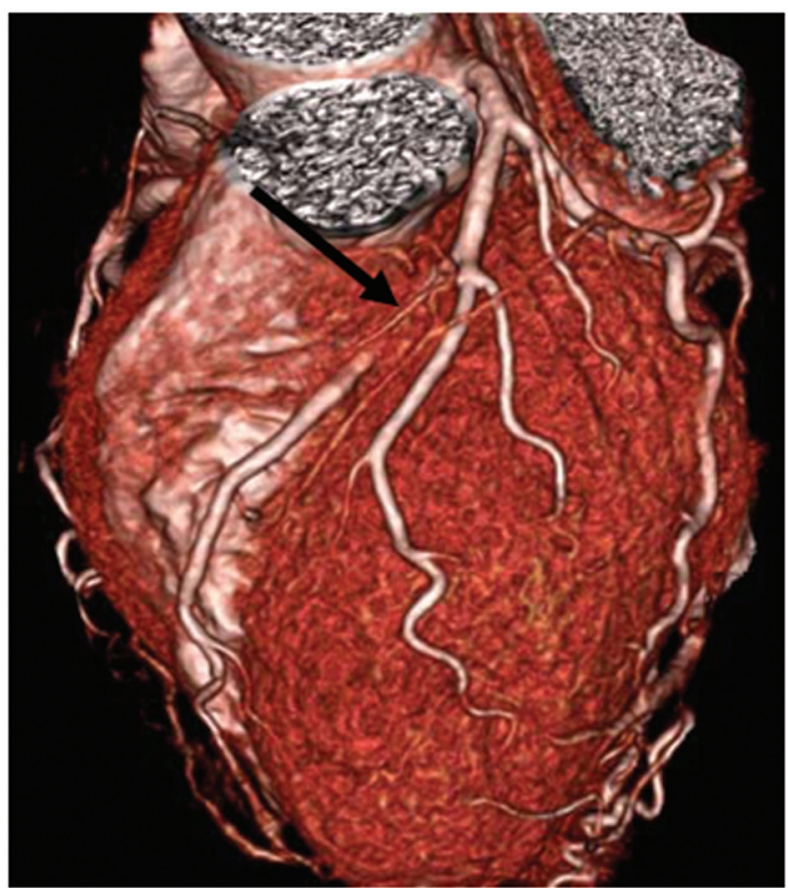

Figura 3. Angiotomografia computadorizada de coronárias: a seta preta indica o segmento da artéria interventricular anterior que se encontra envolvido por feixes de miocárdio. (Fonte: $\mathrm{KO}^{12}$ )
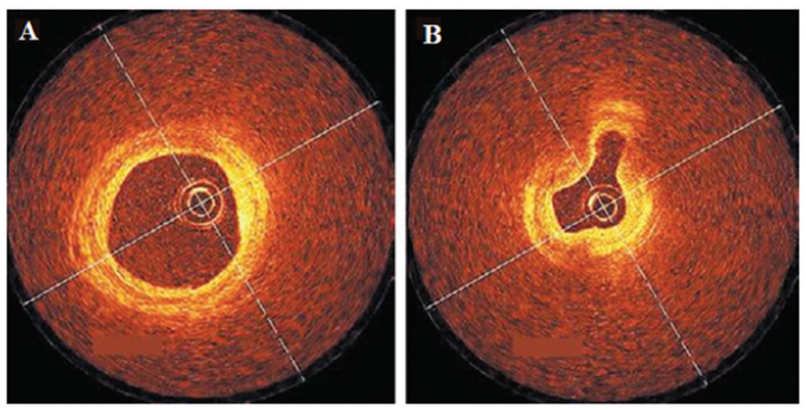

Figura 4. Imagem de tomografia por coerência óptica em indivíduo com ponte miocárdica: não há evidência de doença aterosclerótica, mas há a perviedade do vaso durante a diástole $(A)$ e seu colapso durante a sístole $(B)$. (Fonte: Bose et al. $\left.{ }^{13}\right)$

Ademais, como esta constitui um dos principais diagnósticos diferenciais de síndrome coronariana aguda $^{3}$, é fundamental a avaliação dos achados de exames complementares como eletrocardiograma e marcadores de necrose miocárdica para elucidar o caso e descartar um possível IAM ${ }^{14}$.

\section{Tratamento}

Em pacientes com sintomas angionosos, 
a abordagem inicial preconizada é o tratamento clínico, através do uso de betabloqueador e/ou antagonista de canais de cálcio ${ }^{1,3,7}$, associado a agentes antiplaquetários, visando reduzir sinais/ sintomas de isquemia miocárdica e minimizar o risco de futuros eventos cardíacos adversos. A duração deste tratamento medicamentoso não está bem estabelecida nos diversos estudos acerca o tema ${ }^{3}$.

Os betabloqueadores atuam na redução da frequência cardíaca, no aumento do período da diástole na redução da contratilidade e compressão sistólica do vaso, além da melhora dos sintomas clínicos anginosos. Os antagonistas do canal de cálcio podem ser utilizados na contra indicação do uso de betabloqueadores ${ }^{3}$.

Em caso de persistência da sintomatologia ou com provas funcionais positivas mesmo com o tratamento clínico otimizado instituído, há indicação de intervenção percutânea com uso de stents ou tratamento cirúrgico (miotomia ou revascularização miocárdica) $)^{7}$

Os primeiros relatos sobre abordagem intervencionista através do implante de stent coronariano em PM refratária a terapia medicamentosa foram realizados por Stables et al. ${ }^{1}$, em 1995. O stent não permite que haja a compressão da luz da coronária, minimizando anormalidades do fluxo diastólico, melhorando os sintomas clínicos. Porém, diversos estudos sugerem a necessidade de novas intervenções em cerca de um terço dos casos devido a estenose dos stents ${ }^{3}$.

Antes da realização de intervenção coronariana percutânea, o tratamento de escolha para pacientes em tratamento clínico e com persistência dos sintomas era a miotomia cirúrgica. Com a descompressão cirúrgica do segmento tunelizando, havia melhora dos

\section{REFERÊNCIAS}

1. Möhlenkamp S, Hort W, Ge J, Erbel R. Update on myocardial bridging. Circulation. 2002;106(20):261622.

2. Esteves V, Barbosa RR, Costa Jr JR, Staico R, Feres F, Sousa AGMR, Sousa JE. Infarto agudo do miocárdio associado a ponte miocárdica. Rev Bras Cardiol Invasiva. 2010;18(4):468-72.

3. Pereira AB, Castro DSP, Menegotto ET, Amaral WM, Castro GSP. Ponte Miocárdica: Evolução Clínica e Terapêutica. Arq Bras Cardiol. 2010;94(2):188-94.

4. Lima VJM, Cavalcanti JS, Tashiro T. Pontes de miocárdio e sua relação com o ramo interventricular sintomas de dor torácica e isquemia nos pacientes com compressão severa da artéria interventricular anterior ${ }^{3}$.

Na cirurgia de revascularização do miocárdio, outra opção cirúrgica para tratamento da PM, é realizada uma anastomose entre as artérias torácica interna e interventricular anterior ${ }^{3}$. No entanto, a cirurgia deve ser limitada a pacientes com angina pectoris grave e evidência de isquemia miocárdica clinicamente relevante ${ }^{1}$.

\section{Prognóstico}

Indivíduos com PM geralmente tem um bom prognóstico a longo prazo ${ }^{1}$. Porém, segundo Pereira et al. ${ }^{3}$, a falta de estudos com grupos de pacientes sintomáticos com elevado grau de compressão sistólica e diastólica e evidências de isquemia não permite afirmar com veemência o prognóstico neste grupo específico. Mais estudos precisam ser realizados acerca deste assunto para maior elucidação da PM.

\section{CONSIDERAÇÕES FINAIS}

Embora seja um achado anatômico que geralmente não gere sintomas, em raros casos a PM pode causar complicações importantes, podendo causar inclusive morte súbita.

Portanto, deve-se pensar em PM principalmente naqueles pacientes jovens, com baixo risco para doenças cardiovasculares e sem evidências de isquemia miocárdica, porém apresentando dor torácica, ou então sintomas não condizentes com a severidade da doença arterial coronariana. anterior da artéria coronária esquerda. Arq Bras Cardiol. 2002;79(3):215-8.

5. Smith SC, Taber MT, Robiolio PA, Lasala JM. Acute myocardial infarction caused by a myocardial bridge treated with intracoronary stenting. Cathet Cardiovasc Diagn. 1997;42:209-12.

6. Hayashi T, Ishikawa K. Myocardial bridge: harmless or harmful. Intern Med. 2004;43(12):1097-8.

7. Santos LM, Araújo EC, Sousa LNL. Ponte miocárdica multiarterial: apresentações clínica e anatômica incomuns. Arq Bras Cardiol. 2007;88(4):e73-e75.

8. Abdou M. Myocardial bridging causing ischemia and 
recurrent chest pain: a case report. Int Arch Med. 2011;4:24.

9. Teragawa $\mathrm{H}$, Fukuda $\mathrm{Y}$, Matsuda $\mathrm{K}$, Hisao $\mathrm{H}$, Higashi $\mathrm{Y}$, Yamaguta $\mathrm{T}$, et al. Myocardial bridging increases the risk of coronary spasm. Clin Cardiol. 2003;26(8):377-83.

10.Özlem Soran Ö, Pamir G, Erol Ç, Kocakavak C., Sabah I. The incidence and significance of myocardial bridge in a prospectively defined population of patients undergoing coronary angiography for chest pain. Tokai J Exp Clin Med. 2000;25(2):57-60.

11. Araújo JAR, Fé-Filho NM, Souza FJR, Cruz-Junior A, Rodrigues SS, Silva FAL. Rescue coronary angioplasty in a patient with acute myocardial infarction and myocardial bridge. Arq Bras Cardiol. 1999;72(2):188-90.

12. Ko SG. An overview of myocardial bridging with a focus on multidetector ct coronary angiographic findings. Korean Circ J. 2008;38(11):583-9.

13. Bose D, Phillip S. High-resolution imaging of myocardial bridging. New Engl J Med. 2008;358(4):392.

14. Chansky M, Perez AR, Lima N. Infarto agudo do miocárdio em adulto jovem, portador de ponte miocárdica e artérias coronárias normais. Einstein. 2004;2(3):208-11.

15. Vilallonga JR. Anatomical variations of the coronary arteries: I. the most frequent variations. Eur J Anat. 2003;7:29-41. 